Revista Destaques Acadêmicos, Lajeado, v. 12, n. 3, 2020. ISSN 2176-3070

DOI: http://dx.doi.org/10.22410/issn.2176-3070.v12i3a2020.2653

http://www.univates.br/revistas

\title{
PERFIL NUTRICIONAL E INSEGURANÇA ALIMENTAR DE CRIANÇAS EM VULNERABILIDADE SOCIAL
}

\author{
Patrícia Bergjohann ${ }^{1}$, Patrícia Fassina ${ }^{2}$, Fernanda Scherer Adami ${ }^{3}$
}

Resumo: Introdução: A situação de vulnerabilidade social pode levar a diversas fragilidades e, consequentemente, colocar em risco a saúde e a própria vida dos indivíduos, principalmente, de crianças e adolescentes, além de violar direitos humanos à liberdade, segurança e lazer. Objetivo: Avaliar o estado nutricional, estimar a prevalência de Insegurança Alimentar e associar os indicadores antropométricos, aleitamento materno e nível socioeconômico com a Insegurança Alimentar de crianças participantes do Programa Primeira Infância Melhor (PIM) de três municípios Rio Grande do Sul, Brasil. Método: O estudo foi transversal, com 139 crianças de 0 a 72 meses, inseridas no contexto de vulnerabilidade e integrantes do Programa PIM. Os dados foram obtidos através de entrevistas por dois questionários, a Escala Brasileira de Insegurança Alimentar (EBIA) e um questionário estruturado com questões referentes a indicadores sociais. Os dados relativos a indicadores antropométricos e demais questões sociais foram coletadas no cadastro eletrônico do Programa PIM. Utilizou-se o teste qui quadrado e foi adotado um nível de significância de $p \leq 0,05$. Resultados: O perfil nutricional encontrado, na maioria das crianças, foi eutrofia e $38,8 \%$ apresentaram-se em insegurança alimentar. Verificou-se significância na relação entre escolaridade da mãe e renda mensal com as condições de segurança alimentar e insegurança alimentar ( $\mathrm{p}=0,04$ e $\mathrm{p}=0,025$, respectivamente). Conclusão: A maioria das crianças avaliadas apresentou um perfil nutricional adequado, seguido de sobrepeso, obesidade e magreza, segundo os indicadores antropométricos utilizados neste estudo, assim como a maioria apresentou-se em um contexto familiar de segurança alimentar, seguido de insegurança alimentar de grau leve, moderada e grave.

Palavras-chave: vulnerabilidade social, segurança alimentar, políticas públicas, criança.

1 Nutricionista graduada pela Universidade do Vale do Taquari - Univates.

2 Mestre em Ambiente e Desenvolvimento (Univates) e Docente do Curso de Nutrição da Univates.

3 Doutora em Ambiente e Desenvolvimento (Univates) e Docente do Curso de Nutrição da Univates. 


\section{Introdução}

A situação de vulnerabilidade social pode levar a diversas fragilidades e, consequentemente, colocar em risco a saúde e a própria vida dos indivíduos, principalmente, de crianças e adolescentes, além de violar direitos humanos à liberdade, segurança e lazer. Considerando que a pobreza é uma das principais vulnerabilidades que atinge as famílias brasileiras, em seu maior espectro, pode ser compreendida como a incapacidade de uma pessoa ou domicílio de aproveitar as oportunidades disponíveis nos âmbitos socioeconômicos para melhorar a sua qualidade de vida (ALMEIDA et al., 2016). A fim de minimizar esses efeitos, os serviços de saúde e todas as suas dimensões sociais devem estar em harmonia para o alcance do trabalho em rede, bem como a (re) tomada de vínculos e de planos de ação que busquem, de forma constante, a integralidade da atenção (CORÁ; TRINDADE, 2015).

Tendo em vista a importância de intervenções efetivas no campo da exclusão e vulnerabilidade social, o Instituto de Pesquisa Econômica Aplicada (Ipea), em 2015, instituiu o Índice de Vulnerabilidade Social (IVS), que elenca indicadores do bloco vulnerabilidade social do Atlas de Desenvolvimento Humano (ADH) (ATLAS DO DESENVOLVIMENTO HUMANO NO BRASIL, 2013), os quais servem de suporte para sinalizar o acesso, ausência ou insuficiência de infraestrutura urbana, capital humano, renda e trabalho em porções do território brasileiro. Com base no IVS, a região Sul é a porção do território nacional que apresenta a maior quantidade de municípios na faixa de muito baixa vulnerabilidade social e especificamente, o Rio Grande do Sul (RS) possui 30,2\% de seus municípios inseridos nesta mesma faixa (ATLAS DA VULNERABILIDADE SOCIAL NOS MUNICÍPIOS BRASILEIROS, 2015).

Em se tratando de políticas públicas no âmbito da vulnerabilidade social, estas evidenciaram um grande avanço ao longo da história do Brasil, mas ainda são necessários maiores investimentos em projetos sociais, que contemplem as necessidades básicas dos cidadãos em sua totalidade, e não apenas na ótica emergencial (SANTOS; JULIÃO, 2016). Neste propósito de inserção, permeia a Política Pública do Programa Primeira Infância Melhor (PIM), sendo um Programa Estadual, institucionalizado através da Lei ${ }^{\circ} 12.544$, de 03 de julho de 2006 (RIO GRANDE DO SUL, 2006), pelo Governo do Estado do Rio Grande do Sul. O Programa tem seu objetivo pautado na orientação às famílias em risco e vulnerabilidade social, a partir de sua cultura e experiências, para promover o desenvolvimento integral de suas crianças desde a gestação até os seis anos de idade, faixa etária que compreende a primeira infância, na qual ocorrem o descobrimento de sensações, comportamentos e desenvolvimento cerebral (SECRETARIA ESTADUAL DA SAÚDE, 2016).

O PIM, por pertencer a uma esfera da Saúde Pública, possui relação com a Segurança Alimentar e Nutricional (SAN), que se torna passível de observação através das visitas semanais domiciliares. A SAN é um processo multifatorial, 
atrelado a vários fatores que contribuem para o seu alcance, não relacionado apenas ao acesso à alimentação e renda (AMISTÁ; SILVA, 2015).

Nas áreas diagnosticadas na faixa de alta vulnerabilidade social, os indivíduos podem estar mais suscetíveis a situações de Insegurança Alimentar (IA) e Nutricional, fato que pode estar associado à presença de fatores de risco cardiometabólicos em crianças e adolescentes, em decorrência das más escolhas alimentares ou acesso restrito a alimentação equilibrada. Assim, há uma redução da ingestão de frutas e hortaliças e aumento do consumo de carboidratos refinados e gorduras, associado a uma deficiência de micronutrientes, agravando o estado de saúde e criando uma situação de estresse (ROCHA et al., 2016).

A relação da IA com o estado nutricional das crianças está sob influência da transição nutricional, que compreende a elevada prevalência da obesidade infantil, relacionada com a baixa renda das famílias e um grau reduzido de desenvolvimento humano de um país. A nível mundial, os índices de obesidade infantil são alarmantes e, conforme a Organização Mundial da Saúde (OMS), a prevalência de sobrepeso e obesidade em crianças menores de 5 anos aumentou de $4,8 \%$ para 6,1\% entre 1990 e 2014, passando de 31 milhões para 41 milhões de crianças afetadas nesse período (ORGANIZAÇÃO PAN-AMERICANA DA SAÚDE BRASIL, 2016). Portanto, ressalta-se que os esforços dos órgãos Públicos, das esferas municipais, estaduais e federais, devem atentar-se a programas que promovam orientações profissionais às famílias quanto aos gastos da renda concedida pelo Programa Bolsa Família (PBF) (BRASIL, 2004), por exemplo, qualidade da dieta, atividade física e exposição ao comportamento sedentário (BROYLES et al., 2015).

Diante do exposto, o objetivo do presente estudo foi avaliar o estado nutricional, estimar a prevalência de Insegurança Alimentar e associar os indicadores antropométricos, aleitamento materno e nível socioeconômico com a Insegurança Alimentar de crianças participantes do Programa PIM de três municípios do Rio Grande do Sul, Brasil.

\section{Metodologia}

O estudo possui delineamento transversal, com levantamento de dados primários e secundários da população contemplada pelo Programa PIM de três municípios do Vale do Taquari/RS, Brasil, realizado no período de novembro de 2015 a junho de 2016.

De um total de 211 crianças inseridas no PIM, nos três municípios avaliados, registrou-se a perda de 72 crianças, devido à falta de dados nos cadastros, recusa e/ou analfabetismo de alguns pais/responsáveis na assinatura do Termo de Consentimento Livre e Esclarecido (TCLE) ou ainda pelos pais/responsáveis não se encontrarem em casa no momento da visita, constituindo-se o estudo, por 139 crianças. 
Foram incluídas no estudo, as crianças com até seis anos de idade e que possuíam registro no cadastro eletrônico do Programa. Foram excluídas da amostra primária, as crianças cujos responsáveis não assinaram o TCLE. Dos dados secundários, foram excluídas do estudo, as informações das crianças com algum grau de Síndrome de Down, paralisia cerebral ou qualquer outra condição neurológica diagnosticada clinicamente identificada no cadastro.

Os dados primários foram obtidos através da aplicação do questionário da Escala Brasileira de Insegurança Alimentar (EBIA) e de um questionário estruturado com questões inerentes ao período de Aleitamento Materno Exclusivo (AME), idade da mãe na ocasião do nascimento do filho e peso ao nascer aos pais ou responsável pela criança. O peso ao nascer foi classificado de baixo peso quando < $2500 \mathrm{~g}$; peso insuficiente entre 2500-2999 g; peso adequado entre 3000-3999 g e macrossomia quando peso ao nascer $>4000 \mathrm{~g}$ (BRASIL, 2006). Os questionários com os dados primários foram aplicados por profissionais da Secretaria Municipal de Saúde dos municípios, capacitados pela acadêmica do curso de Nutrição, entre os meses de novembro e dezembro de 2015 no domicílio dos entrevistados. Realizou-se a entrevista de forma individual, sem a presença de outros indivíduos para não haver constrangimentos.

O questionário da EBIA permite mensurar o nível de segurança alimentar (SA) ou IA e trata-se de uma escala validada, traduzida e adaptada dos Estados Unidos para a população brasileira, que valoriza o posicionamento da subjetividade e singularidades de experiências, culminando em um processo decisório por parte do entrevistado (AZEVEDO; RIBAS, 2016). A nível brasileiro, o questionário conta com quatorze questões que se referem ao acesso de alimentos nos últimos três meses (BRASIL, 2006). Porém, no presente estudo, utilizou-se a EBIA original de trinta questões, na qual as famílias foram classificadas de acordo com a seguinte pontuação: as famílias com menores de 18 anos foram categorizadas em SA quando, através do questionário somaram zero pontos; IA leve quando somaram de 1 a 5 pontos; IA moderada quando somaram de 6 a 10 pontos e IA grave mediante a soma entre 11 e 15 pontos. Sendo que os pontos foram adicionados a cada resposta positiva, relativa ao "sim", somados ao final e categorizados conforme descrição acima (SEGALLCORRÊA; MARIN-LEON, 2009).

Os dados secundários foram coletados no período entre os meses de novembro e junho de 2016 pela acadêmica do curso de Nutrição e contemplaram informações que constavam no cadastro eletrônico do PIM das crianças inseridas atualmente no Programa e inativas devido ao limite de idade, mas que ainda possuíam as informações no cadastro. Dentre eles foram coletados dados de peso, altura, idade, gênero, grau de instrução da mãe e renda familiar mensal. A renda familiar mensal foi classificada de acordo com as classes de rendimento do Instituto Brasileiro de Geografia e Estatística (IBGE), até 1/2 salário, mais de 1 / 2 a 1 salário, mais de 1 a 2 salários, mais de 2 a 5 salários, mais 
de 5 a 10 salários, mais de 10 a 20 salários e mais de 20 salários (INSTITUTO BRASILEIRO DE GEOGRAFIA E ESTATÍSTICA, 2016).

A aferição do peso e estatura, informadas no cadastro eletrônico, ocorreu previamente nas Unidades Básicas de Saúde nos três municípios, por profissionais capacitados da área da saúde. As crianças menores de 2 anos foram pesadas em balança pediátrica digital marca $W e l m y^{\circledR}$, com o mínimo de indumentária e na presença da mãe ou responsável. As crianças foram mantidas no centro do prato da balança até a possível leitura do peso no visor da mesma. Para a aferição do comprimento das crianças menores de 2 anos, utilizou-se uma régua antropométrica, na qual as crianças foram deitadas paralelamente à escala numérica, descalças e com a cabeça livre de adereços. A cabeça ficou firmemente apoiada na parte fixa do equipamento, com pescoço reto e olhar no plano de Frankfurt; os ombros ficaram em contato com a superfície na qual estavam deitadas e os braços estendidos ao longo do corpo; pressionou-se cuidadosamente os joelhos para baixo e por fim, levou-se a parte móvel do antropômetro até a planta dos pés para realizar a leitura do valor do comprimento (BRASIL, 2011).

As crianças maiores de 2 anos foram pesadas em balanças mecânicas de plataforma marca Welmy ${ }^{\circledR}$, com o mínimo de indumentária e descalças. As crianças ficaram de costas para a balança, no centro do equipamento, eretas, com os pés juntos e braços ao longo do corpo e foi realizada a leitura do peso. A estatura das crianças maiores de 2 anos foi aferida com o antropômetro vertical acoplado à balança, no qual as crianças foram posicionadas descalças, sem adereços na cabeça, no centro do equipamento e foram mantidas eretas, braços estendidos ao longo do corpo e com a cabeça erguida com olhar para o plano de Frankfurt; as pernas ficaram em paralelo e formaram um ângulo reto com os pés; a parte posterior da cabeça, ombros, nádegas, panturrilhas e calcanhares, na ocasião da aferição da estatura, ficaram encostados no antropômetro (BRASIL, 2011).

$\mathrm{O}$ estado nutricional das crianças menores de 5 anos de idade foi classificado pelo escore-z dos indicadores peso-para-idade $(\mathrm{P} / \mathrm{I})$, peso-paraestatura (P/E), índice de massa corporal-para-idade (IMC/I) e estatura-paraidade (E/I). Semelhantemente, os mesmos indicadores foram utilizados para a classificação do estado nutricional das crianças maiores de 5 anos, excluindo-se apenas o escore-z do indicador P/E. Para a classificação, utilizou-se o Software Anthro versão 3.2.2 para crianças menores de 5 anos e o Software Anthro Plus versão 1.0.4 para crianças maiores de 5 anos.

Para a classificação do indicador $\mathrm{P} / \mathrm{I}$, quando escore- $\mathrm{Z}<-3$, muito baixo peso; quando escore- $Z \geq-3$ e $<-2$, baixo peso; quando escore- $Z \geq-2$ e $\leq+2$, peso adequado; quando $>+2$, peso elevado. Na classificação do indicador E/I, quando escore- $Z<-3$, muito baixa estatura; quando escore- $Z \geq-3$ e $<-2$, baixa estatura; quando escore- $Z \geq-2$ e $>+3$, estatura adequada. Para a classificação do $\mathrm{P} / \mathrm{E}$, quando escore- $Z<-3$, magreza acentuada; quando escore- $Z \geq-3$ e $<-2$, 
magreza; quando $\geq-2$ e $\leq+1$, eutrofia; quando $>+1$ e $\leq+2$, risco de sobrepeso; quando $>+2$ e $\leq+3$, sobrepeso e quando $>+3$, obesidade. Na classificação do IMC $/$ I, quando escore- $Z<-3$, magreza acentuada; quando escore- $Z \geq-3$ e $<-2$, magreza; quando escore- $Z \geq-2$ e $\leq+1$, eutrofia; quando escore- $Z>+1$ e $\leq+2$, risco de sobrepeso; quando escore- $Z>+2$ e $\leq+3$, sobrepeso e quando escore- $Z$ $>+3$, obesidade (WORLD AND HEALTH ORGANIZATION, 2016).

Após a digitação dos dados em planilhas do software Microsoft Excel 2007, os dados foram analisados no pacote estatístico Statistical Package for the Social Science (SPSS) versão 20.0. Foram calculadas as estatísticas indicadas para cada tipo de variável: média e desvio padrão para as variáveis quantitativas paramétricas, mediana e amplitude interquartil para as variáveis quantitativas não paramétricas e frequência absoluta e relativa para as variáveis qualitativas. Teste qui-quadrado ou teste exato de Fisher foi adotado para comparar as características das crianças de acordo com a SA. Regressão logística foi realizada para análise multivariada ajustada para potenciais confundidores, tendo a insegurança alimentar como variável dependente. Foram considerados significativos, os valores de $\mathrm{p}<0,05$.

O presente estudo foi aprovado pelo Comitê de Ética em Pesquisa (COEP) do Centro Universitário Univates, sob o parecer de número 1.154.507. As coletas de dados foram realizadas somente após a assinatura da carta de Anuência pelas Secretarias de Saúde dos municípios e após a assinatura do TCLE pelos pais ou responsáveis. Os resultados obtidos serão de conhecimento dos municípios envolvidos no estudo.

\section{Resultados}

A mediana de idade foi igual a $24,2(13,7-39,0)$ meses, sendo a maioria meninos, $51,8 \%(n=72)$. A idade mediana das crianças no momento do cadastro foi igual a 4,2 (1,2 - 19,4) meses. A renda mensal encontrada entre a maioria das famílias foi de 1 e 5 salários mínimos, sendo $36 \%(n=50)$ com renda entre 1-2 salários mínimos, $36 \%(n=50)$ com renda entre 2 e 5 salários mínimos, seguido de $16 \%(n=22)$ de meio a 1 salário mínimo, $8 \%(n=12)$ da população sem rendimento mensal e $4 \%(n=5)$ com até meio salário mínimo.

A maioria das mães apresentou Ensino Fundamental Incompleto (EFI) $43,2 \%(n=60)$, seguido de 21,5\% ( $n=30)$ de Ensino Médio Incompleto (EMI), $18,9 \%(n=26)$ de Ensino Médio Completo (EMC) e 12,9\% $(n=18)$ de Ensino Fundamental Completo (EFC) e idade média no momento do nascimento dos filhos igual a 26,88 $\pm 7,31$ anos. O tempo mediano de AME foi igual a 4,0 (1,0 $6,0)$ meses.

Os dados antropométricos, classificação do estado nutricional a partir dos diferentes indicadores, renda e escolaridade da mãe das crianças estão apresentados na Tabela 1. 
Tabela 1. Estado nutricional das crianças, gênero, idade, período de aleitamento materno exclusivo, renda mensal familiar e escolaridade da mãe, 2016.

\begin{tabular}{|c|c|}
\hline Característica & Estatística descritiva \\
\hline Peso $(\mathrm{kg})$ & $5,52(4,00-10,52)$ \\
\hline Estatura $(\mathrm{cm})$ & $64,94 \pm 17,01$ \\
\hline $\begin{array}{l}\text { Gênero } \\
\text { Feminino } \\
\text { Masculino }\end{array}$ & $\begin{array}{l}67(48,2) \\
72(51,8)\end{array}$ \\
\hline $\begin{array}{l}\text { Idade das crianças } \\
0-2 \text { meses } \\
2-6 \text { meses }\end{array}$ & \begin{tabular}{|l}
$119(85,6)$ \\
$20(14,3)$ \\
\end{tabular} \\
\hline $\begin{array}{l}\text { Idade da mãe } \\
\leq 16 \text { anos } \\
\text { entre } 16 \text { e } 30 \text { anos } \\
\geq 30 \text { anos }\end{array}$ & $\begin{array}{l}8(5,9) \\
83(61,5) \\
44(32,6)\end{array}$ \\
\hline $\begin{array}{l}\text { Aleitamento materno exclusivo } \\
0 \text { meses } \\
1-3 \text { meses } \\
4-6 \text { meses } \\
>6 \text { meses }\end{array}$ & $\begin{array}{l}23(16,5) \\
49(35,3) \\
61(43,9) \\
6(4,3) \\
\end{array}$ \\
\hline $\begin{array}{l}\text { Peso / idade }(\mathrm{n}=135) \\
\text { Muito baixo peso/Baixo peso } \\
\text { Peso adequado } \\
\text { Peso elevado }\end{array}$ & $\begin{array}{l}24(17,6) \\
103(76,3) \\
8(5,9)\end{array}$ \\
\hline $\begin{array}{l}\text { Estatura / idade }(\mathrm{n}=116) \\
\text { Muito baixa estatura/Baixa estatura } \\
\text { Estatura adequada }\end{array}$ & $\begin{array}{l}44(37,9) \\
72(62,1)\end{array}$ \\
\hline $\begin{array}{l}\text { Peso / estatura }(\mathrm{n}=112) \\
\text { Magreza acentuada/Magreza } \\
\text { Eutrofia } \\
\text { Risco de sobrepeso } \\
\text { Sobrepeso } \\
\text { Obesidade }\end{array}$ & $\begin{array}{l}13(11,7) \\
67(59,8) \\
17(15,2) \\
9(8,0) \\
6(5,4) \\
\end{array}$ \\
\hline $\begin{array}{l}\text { Índice de massa corporal / idade }(\mathrm{n}=116) \\
\text { Magreza acentuada/Magreza } \\
\text { Eutrofia } \\
\text { Sobrepeso } \\
\text { Obesidade/Obesidade Grave }\end{array}$ & $\begin{array}{l}18(15,6) \\
70(60,3) \\
17(14,7) \\
11(9,5)\end{array}$ \\
\hline $\begin{array}{l}\text { Renda mensal } \\
\text { Sem rendimento } \\
\text { Até meio salário mínimo } \\
\text { De meio a } 1 \text { salário mínimo } \\
\text { Entre } 1 \text { e } 2 \text { salários mínimos } \\
\text { Entre } 2 \text { e } 5 \text { salários mínimos }\end{array}$ & $\begin{array}{l}12(8,0) \\
5(4,0) \\
22(16,0) \\
50(36,0) \\
50(36,0)\end{array}$ \\
\hline
\end{tabular}




\begin{tabular}{l|l}
\hline Característica & Estatística descritiva \\
\hline Escolaridade materna & \\
Sem grau de instrução algum & $2(1,4)$ \\
Fundamental Incompleto & $60(43,2)$ \\
Fundamental Completo & $18(12,9)$ \\
Médio Incompleto & $30(21,5)$ \\
Médio Completo & $26(18,9)$ \\
Superior Completo & $2(1,4)$ \\
Superior Incompleto & $1(0,7)$ \\
\hline Classificação EBIA & \\
Segurança Alimentar & $85(61,2)$ \\
Insegurança alimentar leve & $43(30,9)$ \\
Insegurança alimentar moderada & $9(6,5)$ \\
Insegurança alimentar grave & $2(1,4)$ \\
\hline
\end{tabular}

Dados apresentados como média e desvio padrão, mediana e amplitude interquartil ou frequência absoluta ou relativa.

A pontuação mediana da EBIA foi igual a $0,0(0,0-3,25$ pontos), sendo a maioria dos participantes classificados em condições de SA ( $\mathrm{n}=85 ; 61,2 \%)$, $30,9 \%(n=43)$ em condições de IA leve, $6,5 \%(n=9)$ moderada e apenas $1,4 \%$ $(n=2)$ em condições de IA grave.

Os participantes foram agrupados quanto à classificação da EBIA em dois grupos: "em condição de SA" e "em condição de IA", não sendo observada diferença significativa entre os grupos quanto à proporção de meninos e meninas, período de AME e do diagnóstico nutricional avaliado pelos diferentes indicadores nutricionais, mas observou-se diferença significativa entre os grupos quanto à renda familiar mensal $(\mathrm{p}=0,001)$, conforme demonstrado na Tabela 2.

Tabela 2. Características da amostra de acordo com a condição de SA e IA, 2016

\begin{tabular}{l|c|c|c}
\hline \multicolumn{1}{c|}{ Característica } & $\begin{array}{c}\text { Segurança } \\
\text { Alimentar (n=85) }\end{array}$ & $\begin{array}{c}\text { Insegurança } \\
\text { Alimentar (n=54) }\end{array}$ & P \\
\hline $\begin{array}{l}\text { Gênero } \\
\text { Masculino }\end{array}$ & $41(48,2)$ & $31(57,4)$ & $0,303^{1}$ \\
Feminino & $44(51,8)$ & $23(42,6)$ & \\
\hline * AME & $48(56,5)$ & $39(72,2)$ & $0,073^{1}$ \\
$\leq 4$ meses & $37(43,5)$ & $15(27,8)$ & \\
$>4$ meses & $38(44,7)$ & $39(73,6)$ & $0,001^{1}$ \\
\hline Renda mensal & $47(55,3)$ & $14(26,4)$ & \\
até 2 salários mínimos & & & \\
mais de 2 salários mínimos & $43(51,8)$ & $35(64,8)$ & $0,159^{1}$ \\
\hline Grau de instrução materno & $40(48,2)$ & $19(35,2)$ & \\
$\quad$ Ensino fundamental & & & \\
$>$ ensino fundamental completo & & & \\
\hline
\end{tabular}




\begin{tabular}{l|c|c|c}
\hline \multicolumn{1}{c|}{ Característica } & $\begin{array}{c}\text { Segurança } \\
\text { Alimentar (n=85) }\end{array}$ & $\begin{array}{c}\text { Insegurança } \\
\text { Alimentar (n=54) }\end{array}$ & P \\
\hline $\begin{array}{l}\text { Idade materna } \\
\leq 16 \text { anos }\end{array}$ & $5(6,2)$ & $3(5,6)$ & $0,807^{2}$ \\
entre 16 e 30 anos & $48(59,3)$ & $35(64,8)$ & \\
$\geq 30$ anos & $28(34,6)$ & $16(29,6)$ & \\
\hline Peso / idade & $13(16,4)$ & $11(22,5)$ & $0,446^{2}$ \\
Muito baixo peso / baixo peso & $65(82,3)$ & $38(77,5)$ & \\
Peso adequado & $1(1,3)$ & $0(0,0)$ & \\
Peso elevado & $25(34,7)$ & $19(43,1)$ & $0,197^{1}$ \\
\hline Estatura / idade & $47(65,3)$ & $25(56,8)$ & \\
Muito baixa estatura / Baixa estatura & & & \\
Estatura adequada & $6(8,4)$ & $7(17,1)$ & \multirow{2}{*}{$24,217^{1}$} \\
\hline Peso / Estatura & $43(60,6)$ & $24(58,5)$ & \\
Magreza acentuada / magreza & $22(31,0)$ & $10(24,4)$ & \\
$\begin{array}{l}\text { Eutrofia } \\
\text { Risco de sobrepeso / sobrepeso / } \\
\text { obesidade }\end{array}$ & $9(12,5)$ & $9(20,5)$ & $0,511^{1}$ \\
\hline Índice de massa corporal / idade & $46(53,9)$ & $24(54,5)$ & \\
Magreza acentuada / magreza & $17(23,7)$ & $11(25,0)$ & \\
Eutrofia & & & \\
Sobrepeso / Obesidade & & & \\
\hline
\end{tabular}

${ }^{1}$ Teste qui-quadrado. ${ }^{2}$ Teste exato de Fisher.

Dados apresentados em frequência absoluta e relativa.

* AME: Aleitamento Materno Exclusivo.

Considerando-se que a frequência de renda mensal até dois salários mínimos ou maior do que dois salários mínimos foi diferente entre as crianças em condições de segurança e aquelas com insegurança alimentar, a associação entre insegurança alimentar e renda familiar foi melhor explorada em uma análise de regressão logística, tendo a insegurança alimentar como variável dependente e a renda familiar como variável independente. A chance de insegurança alimentar foi cerca de 3,5 vezes maior naqueles com renda mensal até dois salários mínimos em comparação àqueles com renda mensal superior (OR = 3,445; IC95\% 1,635 - 7,261; $\mathrm{p}=0,001)$. A associação permaneceu significativa quando a análise foi ajustada para o grau de instrução da mãe. O aleitamento materno exclusivo não foi associado à insegurança alimentar em análise multivariada (OR = 1,953; IC95\% 0,936 - 4,075; $\mathrm{p}=0,075)$.

\section{Discussão}

A transição nutricional evidenciada na atualidade possui relação direta com alterações demográficas, socioeconômicas e epidemiológicas, além de possuir como principal determinante, uma alimentação sem qualidade. $\mathrm{O}$ contexto atual aponta que, diferentemente de tempos atrás, onde se enfrentavam 
as carências nutricionais e deficiências energético-proteicas, atualmente a preocupação está voltada para o sobrepeso e obesidade, tendo como tendência, as doenças crônicas na vida adulta (FONSECA, 2014).

No que diz respeito aos indicadores antropométricos na população avaliada, a maioria das crianças apresentou eutrofia e, somando-se os percentuais de risco de sobrepeso, sobrepeso e obesidade, estes foram superiores aos percentuais relativos à magreza e baixo peso, resultados semelhantes a um estudo que avaliou o perfil nutricional de crianças no interior paulista, que também encontrou maior percentual associado à eutrofia e, por conseguinte, obesidade, seguido de sobrepeso (AMISTÁ; SILVA, 2015). Esses resultados indicam uma tendência destas populações ao sobrepeso e obesidade em relação à desnutrição, sugerindo que intervenções de prevenção se fazem necessárias em todas as faixas etárias, visando à mudança de comportamento (PIGEOT et al., 2016).

Em relação ao indicador E/I, o estudo evidenciou a maioria das crianças, com estatura adequada para a idade, resultado que pode ser associado à média de peso ao nascer adequada encontrada entre as crianças, visto que a baixa estatura é mais frequente entre os nascidos com déficit de peso (PEDRAZA; SALES; MENEZES, 2016). Neste mesmo contexto, o peso ao nascer também é fator relevante para o indicador P/I nas crianças de até 24 meses, segundo estudo realizado no interior de Campina Grande, na Paraíba, denotando que a prevenção do baixo peso ao nascer constitui-se em um fator que influencia no estado nutricional das crianças (PEDRAZA et al., 2016).

$\mathrm{O}$ indicador de $\mathrm{E} / \mathrm{I}$ também evidenciou estatura adequada para a maior parte das crianças avaliadas segundo as condições de SA e IA. Contudo, as crianças em condição de IA, apresentaram tendência em classificaremse com muito baixa e baixa E/I, fato que pode ser ocasionado pelo déficit na alimentação das crianças, redução da área corporal, o que provavelmente predispõe as crianças a um maior acúmulo de gordura corporal, quando expostas a uma alimentação com alta densidade energética e por consequência, levar à classificação de sobrepeso/obesidade infantil, quando utilizados outros indicadores antropométricos que utilizem o peso (MONTEIRO et al., 2014).

A mediana de peso e média de estatura apresentou relação direta e não significativa com a mediana da EBIA, denotando que, apesar da correlação não significativa do ponto de vista estatístico, a SA está paralelamente associada ao adequado estado nutricional. Por outro lado, segundo estudo realizado com 782 crianças, no município de São Leopoldo, RS, mostrou uma relação inversa entre IA e excesso de peso (VICENZI et al., 2015).

Os indicadores antropométricos de $\mathrm{P} / \mathrm{I}, \mathrm{E} / \mathrm{I}, \mathrm{P} / \mathrm{E}$ e IMC/I não expressaram diferença significativa quando comparados com a condição de $\mathrm{SA}$ ou IA da família, porém é relevante explicitar, que para o indicador de P/I nas duas condições, o maior percentual foi de crianças com peso adequado, refletindo um perfil nutricional compatível com adequação em ambas às 
condições. Para os indicadores P/E e IMC/I se observou um maior percentual de crianças em eutrofia, tanto para as famílias classificadas em SA como para as famílias em condições de IA, havendo uma tendência ao sobrepeso/obesidade em ambas as classificações, corroborando com a teoria da transição nutricional supracitada em vários estudos da atualidade de âmbito nacional e internacional (RAMOS, DUMITH, CÉSAR, 2015; HRUBY; HU, 2015; MENEZES et al., 2014; GUEDES et al., 2013; CARVALHO et al., 2014).

A mediana do tempo de AME encontrada no presente estudo, não atingiu o tempo de AME preconizado pelo Ministério da Saúde do Brasil, de 6 meses (ROCHA, 2016). Dado semelhante foi encontrado em um estudo transversal realizado em São Luiz do Maranhão, no qual $57 \%$ das mães entrevistadas não amamentaram exclusivamente até o sexto mês de vida da criança. E, ao se comparar o tempo de AME com as condições de SA e IA, é notório e cabe salientar que um maior percentual de crianças em condição de IA são amamentadas exclusivamente de 4 a 6 meses e inclusive, por um período superior a 6 meses, sugerindo que isso ocorra devido a alguns fatores que podem favorecer ou restringir a duração do $\mathrm{AME}$, como aspectos biológicos, culturais, assistência à saúde e fatores socioeconômicos (BRASIL, 2015). Nesse sentido, outro estudo realizado em São Miguel do Oeste, Santa Catarina, com 86 crianças em área de vulnerabilidade social, também demonstrou um percentual superior de crianças amamentadas exclusivamente de 4 a 6 meses (RIETH; COIMBRA, 2016) resultados semelhantes aos descritos em outro estudo transversal realizado com 239 crianças entre 48 e 60 meses (TAGLIETTI et al., 2014).

Na população do presente estudo, caracterizada pela vulnerabilidade social, a maioria das crianças pertencia a famílias em condições de SA. Assim como em outro estudo de coorte que avaliou a SA e IA, observou um percentual superior de famílias em AS (CALDEIRA, SOUZA, SOUZA, 2015). É provável que a população do PIM também seja contemplada pelos PTDR, por ser considerada em risco e vulnerabilidade social (SECRETARIA ESTADUAL DA SAÚDE, 2016).

Apesar de não ter-se evidenciado a IA em maior escala como em estudos variados (CABRAL et al., 2014; BEZERRA; PEDRAZA, 2015; ANSCHAU; MATSUO; SEGALL-CORRÊA, 2012; MONTEIRO et al., 2014) é necessário ressaltar, no presente estudo, o percentual de famílias nessa condição, resultado semelhante aos encontrado no município de São Leopoldo, RS, onde o percentual de escolares que pertenciam a famílias em IA foi de $45,1 \%$, demandando a necessidade de políticas públicas abrangentes e com poder para garantir acesso a alimentos de qualidade e em quantidade suficiente, visando o bem-estar da população vulnerável (VICENZI et al., 2015). O nível de IA do atual estudo com população em vulnerabilidade social, constatou um percentual maior ao evidenciado na Pesquisa Nacional por Amostras de Domicílios (PNAD), com aplicação do mesmo questionário EBIA, demonstrando que, no ano de 
2009, de 58.646 domicílios analisados não sendo necessariamente em área de vulnerabilidade, 30,2\% apresentavam IA, sendo 18,7\% de magnitude leve, 6,5\% moderada e $5 \%$ grave. A nível regional foi evidenciado, no mesmo PNAD, que a Região Sul, no mesmo período, de 9.067 pessoas, 18,7\% representaram IA, sendo $13,3 \%$ de grau leve, 3,3\% moderada e 2,1\% IA grave, constituindo-se em um percentual menor de IA em relação ao presente estudo (AQUINO et al., 2014).

A idade materna representa uma variável fundamental na relação com a SA, pois expressa o nível de maturidade e responsabilidade para garantir à criança, acesso aos alimentos de qualidade, na prevenção da desnutrição, sobrepeso e obesidade (VICENZI et al., 2015). Informação que pode justificar o resultado do atual estudo que apontou correlação inversa não significativa entre a média da idade materna e a mediana da escala da EBIA, denotando que, quanto menor a idade materna, maior é a chance de a família apresentar-se em IA e quanto maior a idade materna, menor o risco de IA.

A escolaridade materna é um dos principais fatores que podem influenciar no crescimento e desenvolvimento da criança, cuja afirmação pôde ser observada em estudo realizado com dados da Pesquisa Nacional de Demografia e Saúde da Criança e da Mulher, onde foi encontrada associação entre a baixa escolaridade materna e déficit estatural (INSTITUTO BRASILEIRO DE GEOGRAFIA E ESTATÍSTICA, 2010).

Ao analisar a escolaridade da mãe no presente estudo, verificou-se que a maioria das mães apresentou escolaridade de EFI, semelhante a outros estudos realizados em áreas de vulnerabilidade social, que apresentaram um percentual baixo de escolaridade materna, dificultando a inserção no mercado de trabalho, levando a empregos de baixa remuneração e consequentemente, à IA (BEZERRA; PEDRAZA, 2015; GÉA-HORTA et al., 2016). Referente à escolaridade da mãe em relação à SA e IA, o maior percentual de mães com EFI ficou classificado em IA, assim como as mães sem grau de instrução algum. Por outro lado, na categoria EMC, a maioria das mães esteve em condição de SA, indicando que as mães que pertenciam à condição de IA possuíam significativo menor grau de instrução. Em outro estudo, observou-se a mesma relação, mas sem diferença significativa (BEZERRA, 2014).

Ao se avaliar a renda mensal das famílias, verificou-se um maior percentual de famílias com renda entre 1 e 5 salários mínimos, resultados superiores aos de outro estudo que constatou um maior percentual de famílias com renda mensal entre 1 e 3 salários mínimos (SPERANDIO; PRIORE, 2015) e de um estudo realizado no Piauí com 342 escolares, que encontrou o maior percentual de famílias com renda mensal de menos de 1 salário mínimo (ANSCHAU; MATSUO; SEGALL-CORRÊA, 2012).

Observou-se diferença significativa entre a renda mensal das famílias classificadas em SA e IA, onde a renda das famílias classificadas em IA foi menor em relação às classificadas em SA. Assim como um estudo de revisão 
sistemática que identificou nove estudos que corroboram com o presente estudo no que diz respeito ao binômio IA e menor renda (RIBEIRO et al., 2015). Semelhante a outros estudos realizados com famílias em vulnerabilidade social, que encontraram associação positiva entre IA e menor renda e concluíram que para um cenário ideal de SA, deve haver uma garantia de renda mínima para melhorar as condições de IA (MORAIS et al., 2014; FACCHINI et al., 2014). Diferentemente de outro estudo realizado em Santa Maria com famílias usuárias da atenção primária que não observou diferença significativa entre essas variáveis (BEZERRA, 2014).

Dentre as limitações deste estudo pode-se relatar a confiabilidade dos dados secundários, pela possibilidade de erros de digitação, assim como o delineamento transversal, por não possibilitar o acompanhamento dos fatores avaliados em âmbito temporal.

Deve-se ainda, considerar que a generalização dos resultados deve ser conduzida com precaução, devido ao número da amostra ser representativa apenas para a população com características demográficas e socioeconômicas específicas da região. Sendo assim, o estudo contribui para a validade interna no que diz respeito ao manejo dos resultados encontrados em relação às ações que são conduzidas pelo Programa PIM e demais políticas públicas para a população em vulnerabilidade social, o que deve instigar outros estudos sobre o estado nutricional e IA e demais fatores associados, em outras áreas de vulnerabilidade social com particularidades distintas.

\section{Conclusão}

Concluiu-se que a maioria das crianças apresentou um perfil nutricional adequado, seguido de sobrepeso, obesidade e magreza, segundo os indicadores antropométricos utilizados neste estudo, assim como a maioria apresentouse em um contexto familiar de SA, seguido de IA leve, moderada e grave. Uma maior renda constituiu-se como um determinante para a presença da SA, bem como um maior grau de instrução materno. A correlação inversa encontrada entre a idade materna e a EBIA estabeleceu um maior risco de IA nas situações de idade materna reduzida e a média de AME não atingiu o tempo recomendado pelo Ministério da Saúde do Brasil. Assim, percebeu-se a necessidade de ações dos serviços de saúde e políticas públicas que garantam a SAN em todos os âmbitos da prevenção e promoção da saúde, com o propósito de intervir positivamente nas situações de IA.

\section{Referências}

ALMEIDA, L. C. M. et al. Principais situações de vulnerabilidade social das famílias da microrregião de Ubá, MG. Oikos: Revista Brasileira de Economia Doméstica, v. 27, n. 1, p. 31-58, 2016. 
ANSCHAU, F. R.; MATSUO, T.; SEGALL-CORRÊA, A. M. Insegurança alimentar entre beneficiários de programas de transferência de renda. Revista de Nutrição, v. 25, n. 2, p.177-189, 2012.

AMISTÁ, M. J. M; SILVA, M. V. Estado nutricional de crianças e adolescentes de um município do interior paulista e vínculo com programas de transferência de renda.

Segurança alimentar e nutricional, v. 22, n. 2, p. 721-728, 2015.

ATLAS DA VULNERABILIDADE SOCIAL NOS MUNICÍPIOS BRASILEIROS. Brasília: IPEA, 2015. 77p. Disponível em: <http://ivs.ipea.gov.br/images/ publicacoes/Ivs/publicacao_atlas_ivs.pdf> Acesso em: 01 jul. 2020

ATLAS DO DESENVOLVIMENTO HUMANO NO BRASIL. O Atlas. 2013. Disponível em: <http://www.atlasbrasil.org.br/2013/pt/o_atlas/idhm/> Acesso em: 01 jul. 2020 .

AQUINO, J. S. et al. Insegurança alimentar e perfil socioeconômico, alimentar e nutricional de escolares de áreas urbana e rural do município de Picos, Piauí. Revista de Nutrição, v. 27, n. 4, p. 395-404, 2014.

AZEVEDO, E.; RIBAS, M. T. G. O. Estamos seguros? Reflexões sobre indicadores de avaliação da segurança alimentar e nutricional. Revista de Nutrição, v. 29, n. 2, p. 241251, 2016.

BEZERRA, T. A. (In)segurança alimentar familiar com enfoque na iniquidade social. 2014. 102f. Dissertação (Mestrado) - Mestrado em Saúde Pública, Universidade Estadual da Paraíba, Campina Grande, 2014.

BEZERRA, T. A.; PEDRAZA, D. F. (In)segurança alimentar entre famílias com crianças menores de cinco anos residentes em área de vulnerabilidade social de Campina Grande, Paraíba. Revista de Nutrição, v. 28, n. 6, p. 655-665, 2015.

BRASIL. Lei no 10.836, de 09 de janeiro de 2004. Cria o Programa Bolsa Família e dá outras providências. Diário Oficial [da União]. Brasília, 09 jan. 2004.

BRASIL. Ministério da Saúde. Secretaria de Atenção à Saúde. Departamento de Atenção Básica. Dez passos para uma alimentação saudável: guia alimentar para crianças menores de dois anos: um guia para o profissional da saúde na atenção básica. Brasília, 2015. Disponível em: <http:/ / www.redeblh.fiocruz.br/ media/10palimsa_guia13.pdf> Acesso em: 01 jul. 2020.

BRASIL. Ministério da Saúde. Secretaria de Atenção à Saúde. Departamento de Atenção Básica. Orientações para a coleta e análise de dados antropométricos em serviços de saúde: Norma Técnica do Sistema de Vigilância Alimentar e Nutricional - SISVAN. Brasília, 2011. Disponível em: <http:/ /bvsms.saude.gov.br/bvs/ publicacoes/orientacoes_coleta_analise_dados_antropometricos.pdf $>$ Acesso: 01 jul. 2020. 
BRASIL. Ministério da Saúde. Secretaria de Atenção à Saúde. Departamento de Ações Programáticas Estratégicas. Área Técnica de Saúde da Mulher. Pré-natal e puerpério: atenção qualificada e humanizada - manual técnico. Brasília, 2006. Disponível em: $<$ http://bvsms.saude.gov.br/bvs/publicacoes/manual_pre_natal_puerperio_3ed. pdf $>$ Acesso em: 01 jul. 2020.

BROYLES, S. T. et al. The epidemiological transition and the global chilhood obesity epidemic. International Journal of Obesity Supplements, v. 5, n. Suppl 2, p. S3-S8, 2015

CABRAL, C. S. et al. Segurança alimentar, renda e Programa Bolsa Família: estudo de coorte em municípios do interior da Paraíba, Brasil, 2005-2011. Cadernos de Saúde Pública, v. 30, n. 2, p. 393-402, 2014.

CALDEIRA, K. M. S.; SOUZA, J. M. P.; SOUZA, S. B. Excesso de peso e sua relação com a duração do aleitamento materno em pré-escolares. Revista Brasileira de Crescimento e Desenvolvimento Humano, v. 25, n. 1, p. 89-96, 2015.

CARVALHO, A. T. et al. Situação nutricional de crianças menores de 5 anos em municípios do nordeste brasileiro. Journal of Human Growth and Development, $v$. 24, n. 2, p. 221-227, 2014.

CORÁ, E. J.; TRINDADE, L. M. Intersetorialidade e vulnerabilidade no contexto da educação integral. Educação em Revista, v. 31, n. 4, p. 81-94, 2015.

FACCHINI, L. A. et al. Insegurança Alimentar no Nordeste e Sul do Brasil: magnitude, fatores associados e padrões de renda per capita para redução das iniquidades. Cadernos de Saúde Pública, v. 30, n. 1, p. 161-174, 2014.

FONSECA, L. G. A transição nutricional recente no Brasil. 2014. 19f. Trabalho de Conclusão de Curso [Graduação] - Departamento de Nutrição, Universidade de Brasília, Brasília, 2014.

GÉA-HORTA, T. et al. Associação entre fatores socioeconômicos maternos e resultados nutricionais em crianças menores de 5 anos de idade. Jornal de Pediatria, v. 92, n. 6, p. 574-580, 2016.

GUEDES, D. P. et al. Baixo peso corporal/magreza, sobrepeso e obesidade de crianças e adolescentes de uma região brasileira de baixo desenvolvimento econômico. Revista Paulista de Pediatria, v. 31, n. 4, p. 437-443, 2013.

HRUBY, A.; HU, F. B. The epidemiology of obesity: a big picture.

Pharmacoeconomics, v. 33, n. 7, p. 673-589, 2015.

INSTITUTO BRASILEIRO DE GEOGRAFIA E ESTATÍSTICA. IBGE. Censo Demográfico 2010: trabalho e rendimento. Rio de Janeiro, 2016. Disponível em: <https://ww2.ibge.gov.br/home/estatistica/populacao/censo2010/trabalho_e_ rendimento/default.shtm> Acesso em: 01 jul. 2020. 
INSTITUTO BRASILEIRO DE GEOGRAFIA E ESTATÍSTICA. IBGE. Pesquisa Nacional por Amostra de Domicílios - PNAD. Rio de Janeiro, 2010. Disponível em: <https://www.ibge.gov.br/?id_pesquisa=40> Acesso em: 01 jul. 2020.

MENEZES, C. F. B. et al. Incidence of overweight/obesity in preschool children during a two-year follow-up. Revista de Nutrição, v. 27, n. 3, p. 269-278, 2014.

MONTEIRO, F. et al. Bolsa Família: insegurança alimentar e nutricional de crianças menores de cinco anos. Ciência \& Saúde Coletiva, v. 19, n. 5, p. 1347-1357, 2014.

MORAIS, D. C. et al. Insegurança alimentar e indicadores antropométricos, dietéticos e sociais em estudos brasileiros: uma revisão sistemática. Ciência \& Saúde Coletiva, v. 19, n. 5, p. 1475-1488, 2014.

ORGANIZAÇÃO PAN-AMERICANA DA SAÚDE BRASIL. Relatório da comissão pelo fim da obesidade infantil busca reverter aumento de sobrepeso e obesidade. Brasília, 2016. Disponível em <https: / / www.paho.org/bra/index.php?option=com content\&view=article\&id=4997:relatorio-da-comissao-pelo-fim-da-obesidade-infantilbusca-reverter-aumento-de-sobrepeso-e-obesidade\&Itemid=820> Acesso em: 01 jul. 2020.

PEDRAZA, D. F. et al. Índices antropométricos de crianças assistidas em creches e sua relação com fatores socioeconômicos, maternos e infantis. Ciência \& Saúde Coletiva, v. 21, n. 7, p. 2219-2232, 2016.

PEDRAZA, D. F.; SALES, M. C.; MENEZES, T. N. Fatores associados ao crescimento linear de crianças socialmente vulneráveis do Estado da Paraíba, Brasil. Ciência \& Saúde Coletiva, v. 21, n. 3, p. 935-945, 2016.

PIGEOT, I. et al. Prevention of overweight and obesity in children and adolescents: Critical appraisal of the evidence base. Bundesgesundheitsblatt Gesundheitsforschung Gesundheitsschutz, v. 59, n. 11, p. 1423-1431, 2016.

RAMOS, C. V.; DUMITH, S. C.; CÉSAR, J. A. Prevalence and factors associated with stunting and excess weight in children aged 0-5 years from the Brazilian semi-arid region. Jornal de Pediatria, v. 91, n. 2, p. 175-182, 2015.

RIBEIRO, M. Y. G. et al. Influência da insegurança alimentar no perfil socioeconômico e custos de vida. Revista de Epidemiologia e Controle de Infecção, v. 5, n. 1, p. 01-05, 2015.

RIETH, N. F. A.; COIMBRA, L. C. Caracterização do aleitamento materno em São Luís, Maranhão. Revista de Pesquisa em Saúde, v. 17, n. 1, p. 07-12, 2016.

RIO GRANDE DO SUL. Lei n ${ }^{\circ} \mathbf{1 2 . 5 4 4}$ de 03 de julho de 2006. Institui o Programa Primeira Infância Melhor e dá outras providências. Poder Executivo. Porto Alegre, 03 jul. 2006. 
ROCHA, N. P. et al. Associação de insegurança alimentar e nutricional com fatores de risco cardiometabólicos na infância e adolescência: uma revisão sistemática. Revista Paulista de Pediatria, v. 34, n. 2, p. 225-233, 2016.

ROCHA, S. G. M. O. Obesidade em crianças de região do semiárido brasileiro: tendência temporal e determinantes 2016. 125f. Dissertação (Mestrado) - Programa de Pós-Graduação em Saúde Coletiva, Universidade Federal do Ceará, Fortaleza, 2016.

SANTOS, J. R.; JULIÃO, C. H. O enfrentamento da situação de vulnerabilidade dos adolescentes em uma instituição de atenção social. Revista Família, Ciclos de Vida e Saúde no Contexto Social, v. 4, n. 1, p. 33-39, 2016.

SECRETARIA ESTADUAL DA SAÚDE. O que é o PIM. Porto Alegre, 2016. Disponível em: <http://www.pim.saude.rs.gov.br/site/o-pim/o-que-e/> Acesso em: 01 jul. 2020.

SEGALL-CORRÊA, A. M.; MARIN-LEON, L. A segurança alimentar no Brasil: proposições e usos da Escala Brasileira de Medida da Insegurança Alimentar (EBIA) de 2003 a 2009. Segurança Alimentar e Nutricional, v. 16, n. 2, p. 1-19, 2009.

SPERANDIO, N.; PRIORE, S. E. Prevalência de insegurança alimentar domiciliar e fatores associados em famílias com pré-escolares, beneficiárias do Programa Bolsa Família em Viçosa, Minas Gerais, Brasil. Revista Epidemiologia e Serviços de Saúde, v. 24, n. 4, p. 739-748, 2015.

TAGLIETTI, R. L. et al. Práticas alimentares nos dois primeiros anos de vida: presença de vulnerabilidade em saúde. Scimed: Ciência Baseada na Evidência, v. 24, n. 1, p. 39-45, 2014.

VICENZI, K. et al. Insegurança alimentar e excesso de peso em escolares do primeiro ano no Ensino Fundamental da rede municipal de São Leopoldo, Rio Grande do Sul, Brasil. Cadernos de Saúde Pública, v. 31, n. 5, p. 1084-1094, 2015.

WORLD AND HEALTH ORGANIZATION. Child growth standarts. WHO Anthro (version 3.2.2, January 2011) and macros [online]. Genebra, 2016. Disponível em: <http://www.who.int/childgrowth/software/en/> Acesso em: 01 jul. 2020. 